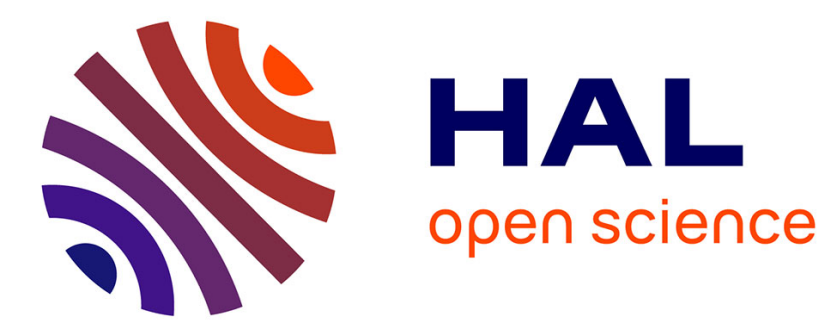

\title{
Determination of set-membership identifiability sets
}

Laleh Hosseini-Ravanbod, Nathalie Verdière, Carine Jauberthie

\section{To cite this version:}

Laleh Hosseini-Ravanbod, Nathalie Verdière, Carine Jauberthie. Determination of set-membership identifiability sets. Mathematics in Computer Science, 2014, 8 (Issue 3-4), pp.391-406. 10.1007/s11786-014-0201-1 . hal-01067127

\section{HAL Id: hal-01067127 \\ https://hal.science/hal-01067127}

Submitted on 23 Sep 2014

HAL is a multi-disciplinary open access archive for the deposit and dissemination of scientific research documents, whether they are published or not. The documents may come from teaching and research institutions in France or abroad, or from public or private research centers.
L'archive ouverte pluridisciplinaire $\mathbf{H A L}$, est destinée au dépôt et à la diffusion de documents scientifiques de niveau recherche, publiés ou non, émanant des établissements d'enseignement et de recherche français ou étrangers, des laboratoires publics ou privés. 


\title{
Determination of set-membership identifiability sets
}

\author{
Laleh Ravanbod, Nathalie Verdière and Carine Jauberthie
}

\begin{abstract}
This paper concerns the concept of set-membership identifiability introduced in [4]. Given a model, a set-membership identifiable set is a connected set in the parameter domain of the model such that its corresponding trajectories are distinct to trajectories arising from its complementary. For obtaining the so-called set-membership identifiable sets, we propose an algorithm based on interval analysis tools. The proposed algorithm is decomposed into three parts namely mincing, evaluating and regularization ([6]). The latter step has been modified in order to obtain guaranteed set-membership identifiable sets. Our algorithm will be tested on two examples.
\end{abstract}

Keywords. Set-membership identifiability, Interval Analysis.

\section{Introduction}

The concept of identifiability is important for insuring that a mathematical model is well-defined in the sense that there exists an unambiguous mapping between its parameters and the output trajectories. This concept insures that parameters estimation procedure will succeed and will give trustable results [14]. However, uncertainties on the knowledge of measures or parameters for example, have to be considered in the numerical procedure. The stochastic framework can take into account these uncertainties but with appropriate assumptions about noise and model error distributions. However, some uncertainties are better characterized by simple bounds. For example, tolerances on parameter values are provided by the manufacturer as lower and upper bounds corresponding to the inherent variability of production line. That is why set-membership (SM) models, in which errors and disturbances affecting a dynamical system are represented by prior bounds, represent an advantageous alternative to stochastic framework. Moreover, success of operational estimation methods developed these last years on SM models confirm their interest, for example [15, 13]. Even theoretical concepts can be treated by interval analysis as the injectivity of a differentiable function. For example, in [9], a numerical algorithm based on interval analysis is presented to determine domains on which a differentiable function is injective and thus, can permit to study the structural identifiability of parametric models. Their algorithms partition the study domain into two domains: an undetermined one on which the function is not proved to be injective and a domain on which the function is injective.

The concept of identifiability of bounded-error uncertain models has been introduced in ([2],[4]). In [4], two definitions named SM and $\mu$-SM-identifiability have been proposed. The first one is purely conceptual whereas the second one subsumes classical identifiability and can be put in correspondence with interval based parameter estimation methods. A SM-identifiable set is a connected

This work was supported by the French National Research Agency (ANR) in the framework of the project ANR-11-INSE-006 (MAGIC-SPS). 
set of the parameter domain such that the trajectories that it generates are distinct to trajectories arising from its complementary. Thus, the search of global ( $\mu$-)SM-identifiable sets permits to determine regions in the parameter space leading to an unique behavior of the corresponding trajectories compared to the rest of parameter space. In practice, it can be important to know if the feasible parameter set is reduced to one connected set or not and in which regions of the parameter space it is located, while taking into account the uncertainties. For example, this knowledge can be useful for doing SM identification and parameter estimation (see for instance [17, 16, 11, 13]) or detection [5]. The aim of this paper is to determine numerically these SM-identifiable connected sets that correspond to different output behaviors for the considered system. For doing this, a method based on differential algebra is proposed. This method consists in linking the $\mu$-SM-identifiability/SM- identifiability definitions to the partial injectivity/restricted-partial injectivity of a real rational function. The definition of partial injectivity has been introduced in [9] and characterizes perfectly the concept of $\mu$-SM-identifiability. We have completed this definition in introducing the notion of restricted-partial injectivity for characterizing the concept of SM-identifiability.

In order to determine numerically these SM-identifiable sets, we have implemented an algorithm composed of three steps ([6]): the mince step, the evaluate step and the regularize step. Indeed, the method consists in calculating the image of the real rational function obtained by the theoretical part. However, it is well-known that it is not always an easy task since interval analysis does not provide any inclusion test for the point test $y \in f(X)$. In [6], they propose three steps in order to obtain a guaranteed inclusion box containing the image of a continuous function. The first step, named mincing consists in building a non-minimal regular subpaving. The second one, the evaluate step, computes the image of a box by the inclusion function. The third one, the regularize step, computes a regularize subpaving containing the image of the inclusion function. However, If we keep their algorithm, the obtained boxes, supposed to correspond to SM-identifiable sets will be composed of non desired parts, that is parts of non SM-identifiable sets. That is why, an algorithm to find a regular internal subpaving is proposed in this step.

The paper is organized as follows. After having recalled the SM-identifiability definitions in the second section, a method based on differential algebra is proposed in order to determine the SMidentifiable sets. This method is based on a proposition linking the $(\mu-) \mathrm{SM}$-identifiability and the (restricted-)partial injectivity of a real rational function. In Section 3, we present our algorithm based on guaranteed numerical computation to find the SM-identifiable sets in the feasible parameters set of a model. In the fourth part, illustrative examples show the efficiency of our algorithm.

\section{The concept of set-membership identifiability}

In this section, we recall the notion of SM-identifiability published in $[4,5]$ for the class of systems formalized by $(2.1)$ :

$$
\Gamma=\left\{\begin{array}{l}
\dot{x}(t, p)=g(x(t, p), u(t), p) \\
y(t, p)=h(x(t, p), p) \\
x\left(t_{0}, p\right)=x_{0} \in X_{0} \\
p \in P \subset \mathcal{U}_{\mathcal{P}} \\
t_{0} \leq t \leq T
\end{array}\right.
$$

where :

- $x(t, p) \in \mathbb{R}^{n}$ and $y(t, p) \in \mathbb{R}^{m}$ denote the state variables and the outputs at time $t$ respectively.

- $u(t) \in \mathbb{R}^{r}$ is the input vector at time $t$.

- the initial conditions $x_{0}$, if any, are supposed to belong to a bounded set $X_{0}$. 
- the vector of parameters $p$ belongs to a connected set $P$ supposed to be included in $\mathcal{U}_{\mathcal{P}}$ where $\mathcal{U}_{\mathcal{P}}$ is an a priori known set of admissible parameters. $\mathcal{U}_{\mathcal{P}}$ is either included in $\mathbb{R}^{p}$ or equal to $\mathbb{R}^{p}$.

- the functions $g$ and $h$ are real and analytic ${ }^{1}$ on $M$, where $M$ is an open set of $\mathbb{R}^{n}$ such that $x(t, p) \in M$ for every $t \in\left[t_{0}, T\right]$ and $p \in P . T$ is a finite or infinite time bound.

Afterwards, $P^{c}$ will design the complementary of $P$ in the considered space.

\subsection{Definitions}

The definitions that are proposed are given in the case of controlled systems but they can be stated similarly in the case of uncontrolled systems. In these definitions, $Y_{\Gamma}(P, u)$ denotes the set of outputs, solution of $\Gamma$ with the input $u$, where $P$ represents a connected set of $\mathbb{R}^{p} . Y_{\Gamma}(P, u)$ is also called the output behavior of $\Gamma$ arising from $P . P^{*}$ is a connected set of $\mathbb{R}^{p}$.

Definition 2.1. The model $\Gamma$ given by (2.1), $P^{*} \neq \emptyset, P^{*} \subseteq \mathcal{U}_{\mathcal{P}}$, is globally SM-identifiable if there exists an input $u$ such that $Y_{\Gamma}\left(P^{*}, u\right) \neq \emptyset$ and $Y_{\Gamma}\left(P^{*}, u\right) \cap Y_{\Gamma}(\tilde{P}, u) \neq \emptyset, \tilde{P} \subseteq \mathcal{U}_{\mathcal{P}} \Longrightarrow P^{*} \cap \tilde{P} \neq \emptyset$.

The previous definition expresses that a connected set $P^{*}$ is globally SM-identifiable if the output behavior of $\Gamma$ arising from $P^{*}$, i.e. the output behavior of $\Gamma$ for any $p \in P^{*}$, is distinguishable from the output behavior of $\Gamma$ arising from its complementary set $P^{* c}$, i.e. $Y_{\Gamma}\left(P^{*}, u\right)$ and $Y_{\Gamma}\left(P^{* c}, u\right)$ do not share any identical output trajectory.

Let us now consider a nonempty bounded connected set $\Pi$ of $\mathbb{R}^{p}$ and $d$ a classical metric on $\mathbb{R}^{p}$ [1], [12]. On the metric space $(\Pi, d)$, let $\mu$ be a continuous map from $\Pi$ to $\Pi$. $\mu$ is a contraction if there is a nonnegative number $k<1$ such that for all $\pi_{1}, \pi_{2}$ in $\Pi, d\left(\mu\left(\pi_{1}\right), \mu\left(\pi_{2}\right)\right)<k d\left(\pi_{1}, \pi_{2}\right)$ [12]. Let us also define the diameter of $\Pi$ by the least upper bound of $\left\{d\left(\pi_{1}, \pi_{2}\right), \pi_{1}, \pi_{2} \in \Pi\right\}$.

In the following definition, the set $P^{*}$ is supposed to be a bounded connected set and $\mu$ is a contraction from $P^{*}$ to $P^{*}$.

Definition 2.2. The nonempty bounded connected set $P^{*} \subseteq \mathcal{U}_{\mathcal{P}}$ is globally $\mu$-SM-identifiable if, for all contractions $\mu$ from $P^{*}$ to $P^{*}, \mu\left(P^{*}\right)$ is globally SM-identifiable.

Under the conditions of definition 2.2, we may equivalently say that the model $\Gamma$ given by $(2.1)$ is globally $\mu$-SM-identifiable with respect to $P^{*}$.

Definition 2.2 differs from definition 2.1 in the sense that the set $P^{*}$ may be reduced as small as desired by the contraction $\mu$ while still retaining the property of SM-identifiability. This is true by Banach fixed-point theorem, which implies that the diameter of $\mu\left(P^{*}\right)$ tends to zero [12]. In this case, $\mu$-SM-identifiability meets classical identifiability and, interestingly, it means that classical identifiability holds for any $p \in P^{*}$. In [4], it has been proven that the $\mu$-SM-identifiability is equivalent to the classical identifiability (see [10] for a good survey of this definition), that the $\mu$-SM-identifiability implies the global SM-identifiability but the reciprocal is not true.

In Figure 1 , the sets $A_{i}, i=1, \ldots, 5$ are $\mathrm{SM}$-identifiable and our aim is to determine $\mathbb{F}=\left\{F_{0}=\right.$ $\left.A_{0}, F_{1}=A_{1}, F_{2}=A_{3}\right\}$. This list constitutes a list of SM-identifiable sets such that the trajectories generated by these connected sets are distinct to their complementary.

\subsection{Partial injectivity}

The definition of partial injectivity of a function has been introduced in [8]. This notion perfectly characterizes $\mu$-SM-identifiability. A second definition named restricted-partial injectivity completes the first one and characterizes the global SM-identifiability.

Definition 2.3. Consider a function $f: \mathcal{A} \rightarrow \mathcal{B}$ and any set $\mathcal{A}_{1} \subseteq \mathcal{A}$. The function $f$ is said to be a partial injection of $\mathcal{A}_{1}$ over $\mathcal{A}$, noted $\left(\mathcal{A}_{1}, \mathcal{A}\right)$-injective, if $\forall a_{1} \in \mathcal{A}_{1}, \forall a \in \mathcal{A}$,

$$
a_{1} \neq a \Rightarrow f\left(a_{1}\right) \neq f(a)
$$

\footnotetext{
${ }^{1}$ This assumption is important at section 2.3 for the use of differential algebra.
} 


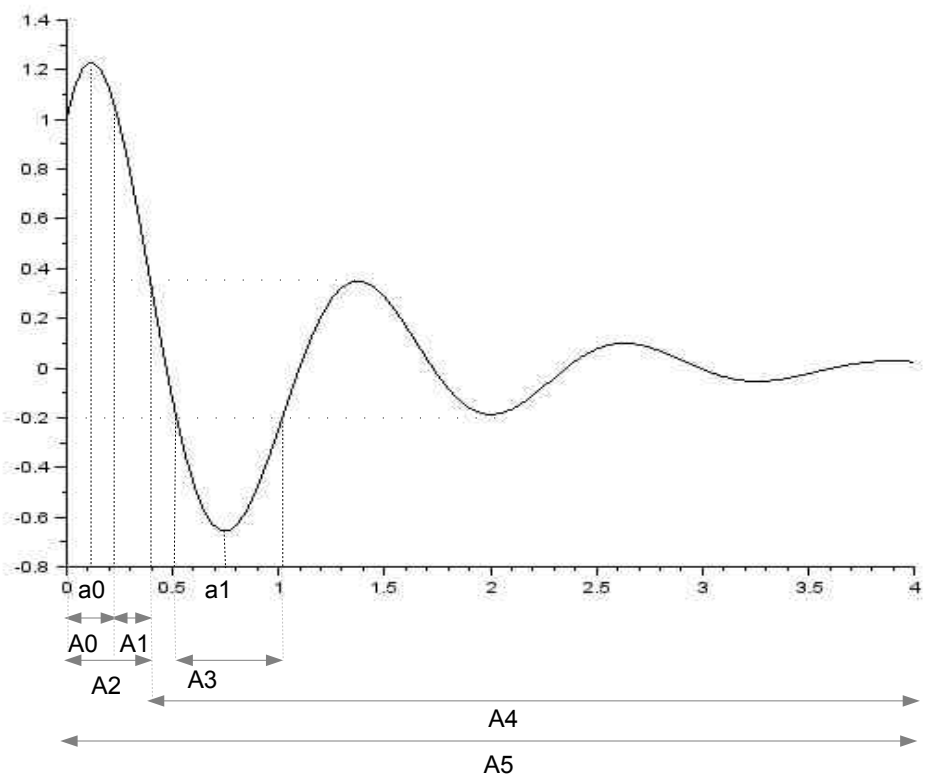

FIGURE 1. Example of SM-identifiable sets

$f$ is said to be $\mathcal{A}$-injective if it is $(\mathcal{A}, \mathcal{A})$-injective.

In [9], an algorithm based on interval analysis for testing the injectivity of a given differentiable function is presented and a solver called ITVIA (Injectivity Test Via Interval Analysis) implemented in $\mathrm{C}++$ is mentioned. From a given function, the solver partitions a given box into two domains: a domain on which the function is partially injective and an indeterminate domain on which the function may or not be injective. When the latter is empty, the function is injective over the initial box.

In order to characterize the global SM-identifiability, the notion of restricted-partial injectivity is introduced. The algorithm proposed in [8], can be easily adapted for testing this new definition. In this definition, $\mathcal{A}_{1}^{c}$ designs the complementary of $\mathcal{A}_{1}$ in $\mathcal{A}$.

Definition 2.4. Consider a function $f: \mathcal{A} \rightarrow \mathcal{B}$ and any set $\mathcal{A}_{1} \subseteq \mathcal{A}$. The function $f$ is said to be a restricted-partial injection of $\mathcal{A}_{1}$ over $\mathcal{A}_{1}^{c}$ or a $\left(\mathcal{A}_{1}, \mathcal{A}\right)$-restricted injection if :

$$
\forall a_{1} \in \mathcal{A}_{1}, \forall a \in \mathcal{A}_{1}^{c}, f\left(a_{1}\right) \neq f(a) .
$$

In section 3, the algorithm proposed in [9] is taken again and adapted to determine the non $\mu$-SM-identifiable parameters domains.

\subsection{Method for analyzing the ( $\mu$-)SM-identifiability of a connected set}

In $[4,15]$, we have proposed two methods for testing the global ( $\mu$-)SM-identifiability definition: the first one is based on the Taylor Series approach, the second one on differential algebra. The latter presents many advantages. The first one is that it reduces the SM- identifiability study of the model to the study of a real rational function whose range is the parameter domain of the model. The second one is that this approach leads to a numerical method given a first estimation of the parameters of the model $([14,15])$. In $[4,15]$, equivalent conditions between the definition of $\mu$-SM-identifiability and the injectivity of a real rational function has been proved and is recalled below. In this paper, we present an equivalent condition between the definition of SM-identiability and the restricted-partial injectivity of the same real rational function. 
The differential algebra [7] permits to obtain differential polynomials linking outputs, inputs and parameters which can be expressed as [14]:

$$
w_{i}(y, u, p)=m_{0}^{i}(y, u)+\sum_{k=1}^{n_{i}} \theta_{k}^{i}(p) m_{k}^{i}(y, u), i=1, \ldots, m,
$$

where $\left(\theta_{k}^{i}(p)\right)_{1 \leq k \leq n_{i}}$ are rational in $p, \theta_{u}^{i} \neq \theta_{v}^{i}(u \neq v),\left(m_{k}^{i}(y, u)\right)_{0 \leq k \leq n_{i}}$ are differential polynomials with respect to $y, u$ and $m_{0}^{i}(y, u) \not \equiv 0$.

$\left\{\theta_{k}^{i}(p)_{1 \leq k \leq n_{i}}\right\}$ is called the exhaustive summary of $w_{i}$.

The size of the system is the number of outputs. For the time being, we suppose that $i=1$, that is there is one output and $n_{1}=n, w_{1}=w, m_{k}^{1}(y, u)=m_{k}(y, u)$. The case of fewer outputs will be seen at the end of this section.

Consider $t_{0}^{+}$the right limit of $t_{0}^{2}$ and $l$ the higher order derivative of $y$ in (2.2). Hereafter, $\Delta w(y, u)$ will design the functional determinant formed from the $\left\{m_{k}(y, u)\right\}_{1 \leq k \leq n}$. The following theorem permits to obtain necessary and sufficient conditions for having global SM-identifiability or $\mu$-SM-identifiability.

Theorem 2.5. Assume that the functional determinant $\Delta w(y, u)$ is not identically equal to zero. Consider $P^{*}$ a connected subset of $\mathcal{U}_{\mathcal{P}}$.

If the function $\phi: p=\left(p_{1}, \ldots, p_{p}\right) \mapsto\left(\theta_{1}(p), \ldots, \theta_{n}(p), y\left(t_{0}^{+}, p\right), \ldots, y^{(l-1)}\left(t_{0}^{+}, p\right)\right)$ is $\left(P^{*}, \mathcal{U}_{\mathcal{P}}\right)$ restricted injective (resp. $\left(P^{*}, \mathcal{U}_{\mathcal{P}}\right)$-injective) then $P^{*}$ is globally $S M$-identifiable (resp. $\mu$-SM-identifiable). In the two cases, if the coefficient of $y^{(l)}$ in (2.2) is not equal to 0 at $t_{0}$, then the reciprocal is valid ${ }^{3}$.

Proof - The proof is an easily extension of the one proposed in [4].

Remark - In the case of $m$ outputs, the procedure is the following. For each of the $m$ obtained differential polynomials $w_{i}(y, u, p)$, the functional determinant is evaluated. If it is not identically equal to zero, the associated exhaustive summary is added to the image of the function $\phi$ whose the (restricted-)partial injectivity has to be studied.

\subsection{Testing the SM-identifiability}

The steps for proving the SM-identifiability's definitions are summed up below.

1. Finding the differential polynomials $w_{i}$ in using, for example, the package DifferentialAlgebra of Maple.

2. Evaluate the functional determinants and construct the function $\phi$.

3. Verify the (restricted-)partial injectivity of the function $\phi$.

The proposed method consists in reducing the study of SM-identifiability's definitions to the study of the (restricted)-partial injectivity of the function $\phi$ whose range corresponds to the admissible parameter set of the model. The domains on which the function is restricted-partial injective but not partial injective will correspond to global SM-identifiable sets and domains on which the function $\phi$ is partially injective will correspond to $\mu$-SM-identifiable sets.

In the following section, an algorithm to determine the SM-identifiable sets in the parameter space is proposed. It is based on interval analysis tools whose main advantage is that it guarantees numerical solutions provided as sets.

\footnotetext{
${ }^{2} t_{0}^{+}$is considered to ensure the existence of derivatives.

${ }^{3}$ When initial conditions are not considered, the function $\phi$ becomes $\phi: p=\left(p_{1}, \ldots, p_{p}\right) \mapsto\left(\theta_{1}(p), \ldots, \theta_{n}(p)\right)$ and the reciprocal of the theorem is not yet valid.
} 


\section{Determination of the ( $\mu$-)SM-identifiable sets}

The proposed algorithms find the connected sets in the range of a continuous differentiable function such that, for each of them, their image is distinct to the one obtained from their complementary in the parameter space. Afterwards, they will be named SM-identifiable sets to be put in correspondence with the SM-identifiable sets defined from the model $\Gamma$. It takes back the algorithm of Lagrange et al. ([8] [9]) called ITVIA (for Injectivity Test Via Interval Analysis) which partitions the domain of a differentiable function into two domains: an undetermined domain not proved partially injective and a partial injective domain. The latter corresponds to a list of $\mu$-SM-identifiable sets. To complete the partition, we have to find the SM-identifiable sets in the undetermined domain.

Denote $f: \mathbb{R}^{n} \rightarrow \mathbb{R}^{m}\left(f \in \mathcal{C}^{1}\right)$ a differentiable vector function defined over a given $n$-dimensional box $[x] \in \mathbb{R}^{n}$, where $\mathbb{I} \mathbb{R}$ is the set of all intervals and $[f]$ its inclusion function [6]. Regarding to [3], a subpaving of $[x]$ is a set of non-overlapping boxes included in $[x]$. A subpaving can be considered either as a collection (list) of boxes $\mathbb{K}=\left\{[x]^{(1)},[x]^{(2)}, \ldots\right\}$ or as a union $\mathbb{K}=[x]^{(1)} \bigcup[x]^{(2)} \bigcup \ldots$. Hence, a subpaving can either be viewed as discrete subset of $\mathbb{I R}^{n}$ or as a convex subset of $\mathbb{R}^{n}$. Subpavings permit to approximate convex sets with arbitrary precision. A regular subpaving is a subpaving generated by successive bisections and it can be easily represented by a binary tree.

After obtaining a paving of $\mu$-SM-identifiable sets by the algorithm ITVIA, we determine its complementary and, to complete the work, we proceed as in Image evaluation [6]. Firstly, we consider a subpaving with boxes of width smaller than $\epsilon$ (mince step). Secondly, we find SM-identifiable sets as a list of non-overlapped boxes (evaluate step). Finally, we transform this list to a regular subpaving (regularize step).

We begin by the case $m=1$ and then we generalize our algorithm to the multidimensional case.

\subsection{Case of unidimensional functions}

Let $\{\mathcal{P}\}$ a collection (list) of non overlapped boxes included in $[x]$, we introduce the following notations. $N$ corresponds to the length of the list $\{\mathcal{P}\} . \max _{[f],\{\mathcal{P}\}}$ (resp. $\min _{[f],\{\mathcal{P}\}}$ ) denotes the sequence of the maximal value (resp. the minimal value) of $[f]$ on each box of $\{\mathcal{P}\}$ and the two following, $\mathcal{M a x}_{[f],\{\mathcal{P}\}}{ }^{+}$and $\mathcal{M i n}_{[f],\{\mathcal{P}\}}{ }^{+}$(resp. $\mathcal{M a x}_{[f],\{\mathcal{P}\}}{ }^{-}$and $\mathcal{M i n}_{[f],\{\mathcal{P}\}}{ }^{-}$), the maximal and minimal values of the sequence $\max _{[f],\{\mathcal{P}\}}$ (resp. $\min _{[f],\{\mathcal{P}\}}$ ). Then, $i_{\mathcal{M} a x}$ and $i_{\mathcal{M} \text { in }}$ are the minimal corresponding indexes. Finally, $\epsilon$ is the maximal diameter of the boxes considered in the list $\{\mathcal{P}\}$. Mathematically, these definitions can be rewritten under the form:

- length $(\{\mathcal{P}\})=N$,

- $\max _{[f],\{\mathcal{P}\}}=\left\{\max \left([f]\left(\{\mathcal{P}\}_{i}\right)\right), 1 \leq i \leq N\right\} \in \mathbb{R}^{N}$,

- $\min _{[f],\{\mathcal{P}\}}=\left\{\min \left([f]\left(\{\mathcal{P}\}_{i}\right)\right), 1 \leq i \leq N\right\} \in \mathbb{R}^{N}$,

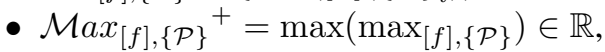

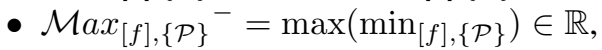

- $\mathcal{M i n}_{[f],\{\mathcal{P}\}^{-}}=\min \left(\min _{[f],\{\mathcal{P}\}}\right) \in \mathbb{R}$,

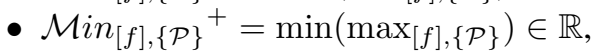

- $\epsilon=w(\{\mathcal{P}\})_{i=1, \ldots, N}=\max _{1 \leq i \leq n}\left(w\left(\{\mathcal{P}\}_{i}\right)\right)$ where $w$ represents paving width. ${ }^{4}$

\section{Example 1:}

In order to illustrate these notations, the following function $f$ defined by:

$$
f(x)=\sin \left(10(x-0.1)^{2}\right) / x, \text { where }[x] \in[0.1,2],
$$

is represented at Figure 2. $\{\mathcal{P}\}$ consists of equal boxes of width $\epsilon=0.05$ in $[0.1,2]$.

${ }^{4}$ If $[x]=[\underline{x}, \bar{x}]$ then $w([x])=\bar{x}-\underline{x}$. 


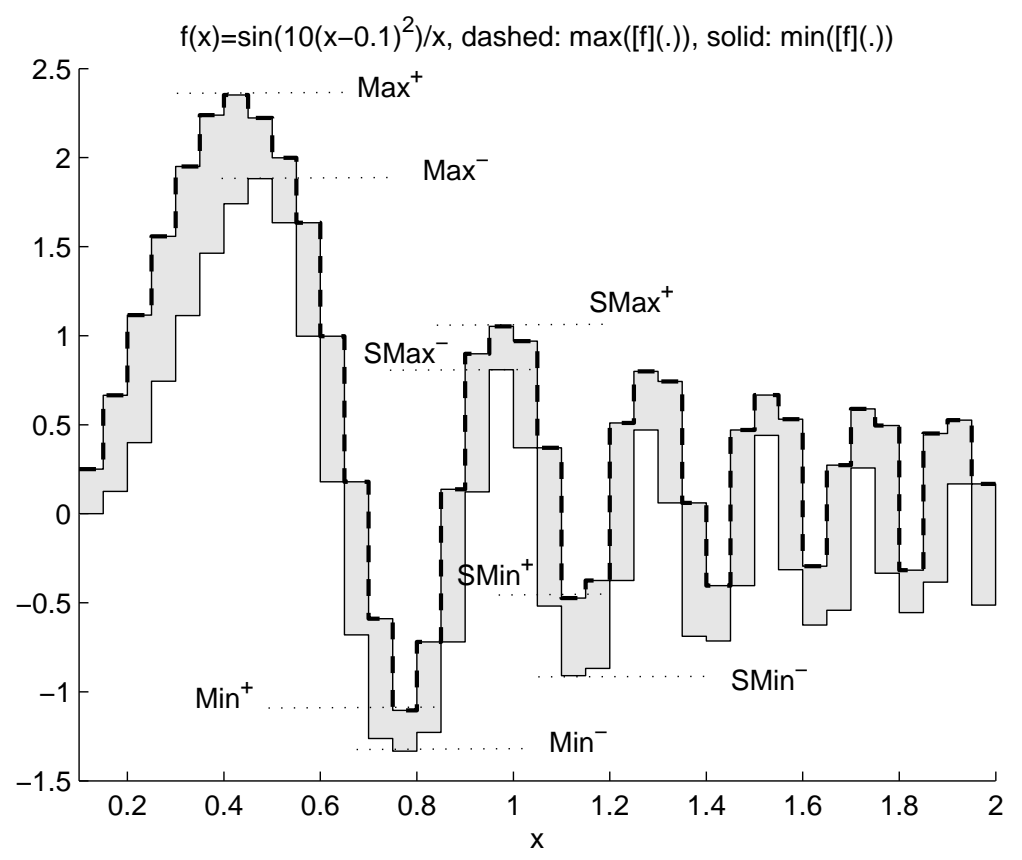

FIGURE 2. Illustration of the notations, to simplify, the subscript $\{[f],\{\mathcal{P}\}\}$ is omitted.

3.1.1. Second maximum and second minimum. The aim of the two following sections is to isolate the domain around the maximum (resp. minimum) of $f$ which constitutes a SM-identifiable set as the interval $[0.2 ; 0.6]$ at Figure 2. For doing this, one has to determine first the second maximum of $f$ (resp. minimum), in particular the lower and the upper values of the box containing this second maximum (resp. minimum). The lower value will be denoted $\mathcal{S} \mathcal{M a x}_{[f],\{\mathcal{P}\}}{ }^{-}$and the upper value, $\mathcal{S} \operatorname{Max}_{[f],\{\mathcal{P}\}}{ }^{+}$(see Figure 2).

While $\mathcal{M a x}_{[f],\{\mathcal{P}\}^{+}}$and $\mathcal{M a x}_{[f],\{\mathcal{P}\}}{ }^{-}$can be directly evaluated from $\max ([f](\{\mathcal{P}\}))$, we propose Algorithm 1 for evaluating $\mathcal{S} \mathcal{M a x}{ }_{[f],\{\mathcal{P}\}}{ }^{+}$or $\mathcal{S M a x}_{[f],\{\mathcal{P}\}}{ }^{-}$. There, $\mathcal{S} \mathcal{M a x}_{[f],\{\mathcal{P}\}}{ }^{+}$and $\mathcal{S} \mathcal{M a x}_{[f],\{\mathcal{P}\}}{ }^{-}$are computed in replacing $M \in \mathbb{R}^{N}$ by $\max ([f](\{\mathcal{P}\}))$ and $\min ([f](\{\mathcal{P}\}))$, respectively. For example, consider that $M=\max ([f](\{\mathcal{P}\}))$.

In this algorithm, we find $\mathcal{M a x}_{[f],\{\mathcal{P}\}}{ }^{+}$, that is the maximum value of the list $\max ([f](\{\mathcal{P}\}))$ and we initialize a list named $\{\mathcal{L}\}$ by the corresponding box of $\mathcal{M a x}_{[f],\{\mathcal{P}\}}{ }^{+}$in $\{\mathcal{P}\}$. Then, we find the next maximum value of $\max ([f](\{\mathcal{P}\}))$. If its corresponding box $[x]_{\text {temp }}$ in $\{\mathcal{P}\}$ is connected to one of the component of $\{\mathcal{L}\}$, that is it intersects one of the box of $\{\mathcal{L}\}$ (see [6] for the definition of connected box), we add it to $\{\mathcal{L}\}$ and we continue. If there exists a box $[x]_{\text {temp }}$ not connected with one of the box of $\{\mathcal{L}\}$, one gets the box containing the second maximum of the function $f$. The second maximum is named disconnected afterwards, an example is shown at Figure 2. If all the boxes $[x]_{\text {temp }}$ are connected with one of the box of $\{\mathcal{L}\}$ then the second maximum is found at the boundary, i.e. at $\{\mathcal{P}\}_{1}$ or $\{\mathcal{P}\}_{N}$.

The same work can be done in order to find $\mathcal{S M i n}_{[f],\{\mathcal{P}\}}{ }^{+}$and $\mathcal{M i n}_{[f],\{\mathcal{P}\}}{ }^{-}$. 


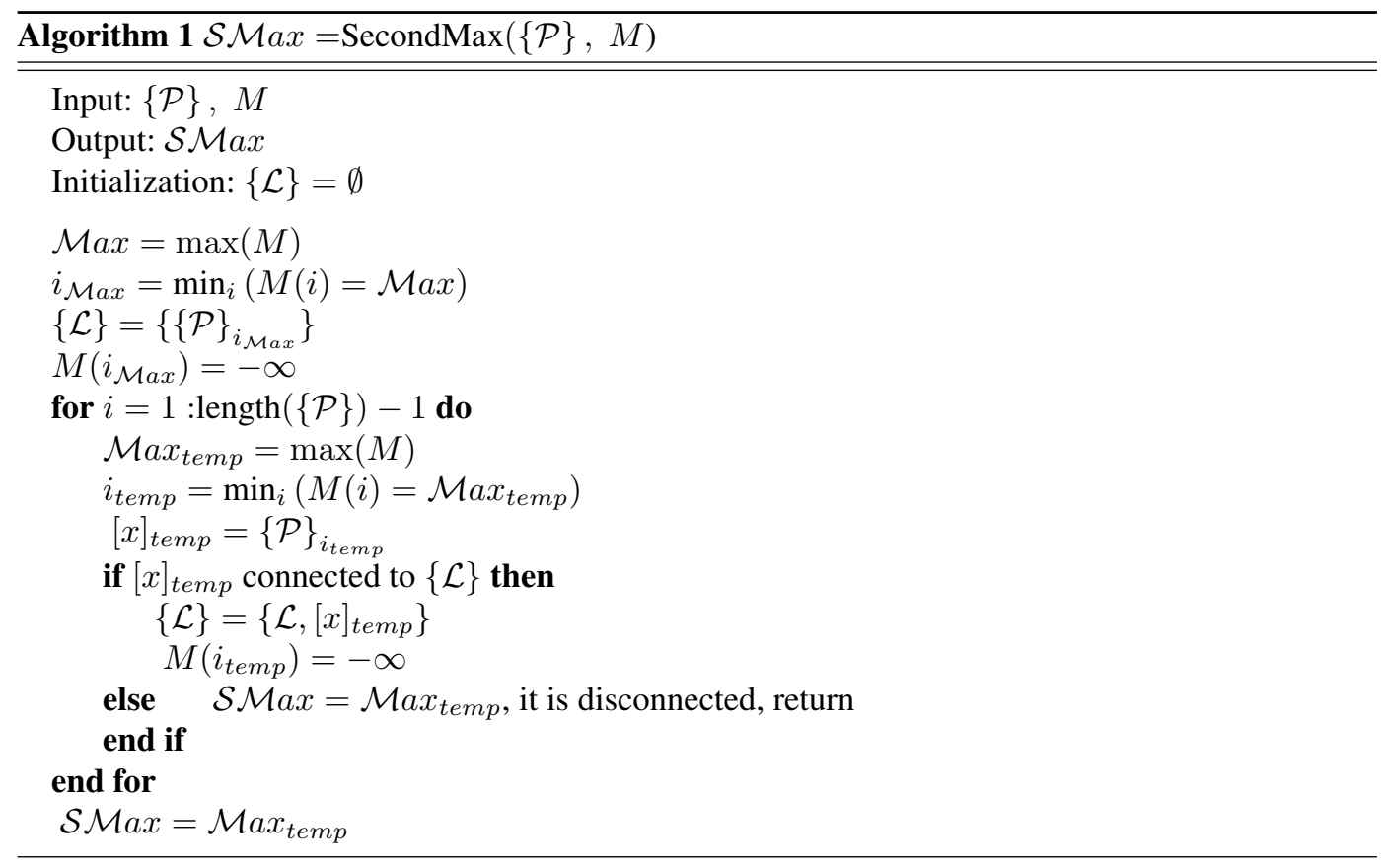

3.1.2. Relation between partitioning and second maximum, minimum. For the final step, we construct the SM-identifiable set defined around the maximum or minimum value of $f$. Indeed, when we search the second maximum or minimum, we obtain the list of boxes $\{\mathcal{L}\}$ whose image, by the function $f$, is not connected with its complementary. However, we are not insure to obtain a connected set. For being convinced, see for example the second figure of Figure 3. Owing to the third box, we do not obtained a connected set. First, let us define the list of boxes $\{\mathcal{L}\}_{[f],\{\mathcal{P}\}, a}^{+}$ (resp. $\{\mathcal{L}\}_{[f],\{\mathcal{P}\}, a}^{-}$) such that their image by the inclusion function has their minimum upper (resp. maximum lower) than $a$.

Definition 3.1. The list of boxes $\{\mathcal{L}\}_{[f],\{\mathcal{P}\}, a}^{+}$is defined by:

$$
\{\mathcal{L}\}_{[f],\{\mathcal{P}\}, a}^{+}=\left\{\{\mathcal{P}\}_{i}, \min \left([f]\left(\{\mathcal{P}\}_{i}\right)\right) \geq a\right\}
$$

Definition 3.2. The list of boxes $\{\mathcal{L}\}_{[f],\{\mathcal{P}\}, a}^{-}$is defined by:

$$
\{\mathcal{L}\}_{[f],\{\mathcal{P}\}, a}^{-}=\left\{\{\mathcal{P}\}_{i}, \max \left([f]\left(\{\mathcal{P}\}_{i}\right)\right) \leq a\right\} .
$$

The notion of connected lists formed of intervals is introduced before giving a sufficient condition for having a connected SM-identifiable set in $[x]$.

Definition 3.3. A list $\{\mathcal{L}\}$ formed of intervals is said connected if the union of its components is an interval of $\mathbb{R}$.

\section{Proposition 3.4.}

In (3.2), for $a=\mathcal{S} \mathcal{M a x}_{[f],\{\mathcal{P}\}}{ }^{+}$, if the list $\{\mathcal{L}\}_{[f],\{\mathcal{P}\}, a}^{+}$is a connected set, then it is a SM-identifiable set. We denote it by $\{\mathcal{L}\}_{S M I,[f],\{\mathcal{P}\}}^{+}$.

Proof - The list $\{\mathcal{L}\}_{[f],\{\mathcal{P}\}, a}^{+}$corresponds, by construction, to the list of boxes not having a common intersection with its complementary. Concretely, the image $\{\mathcal{L}\}_{[f],\{\mathcal{P}\}, a}^{+}$of $f$ is distinct 

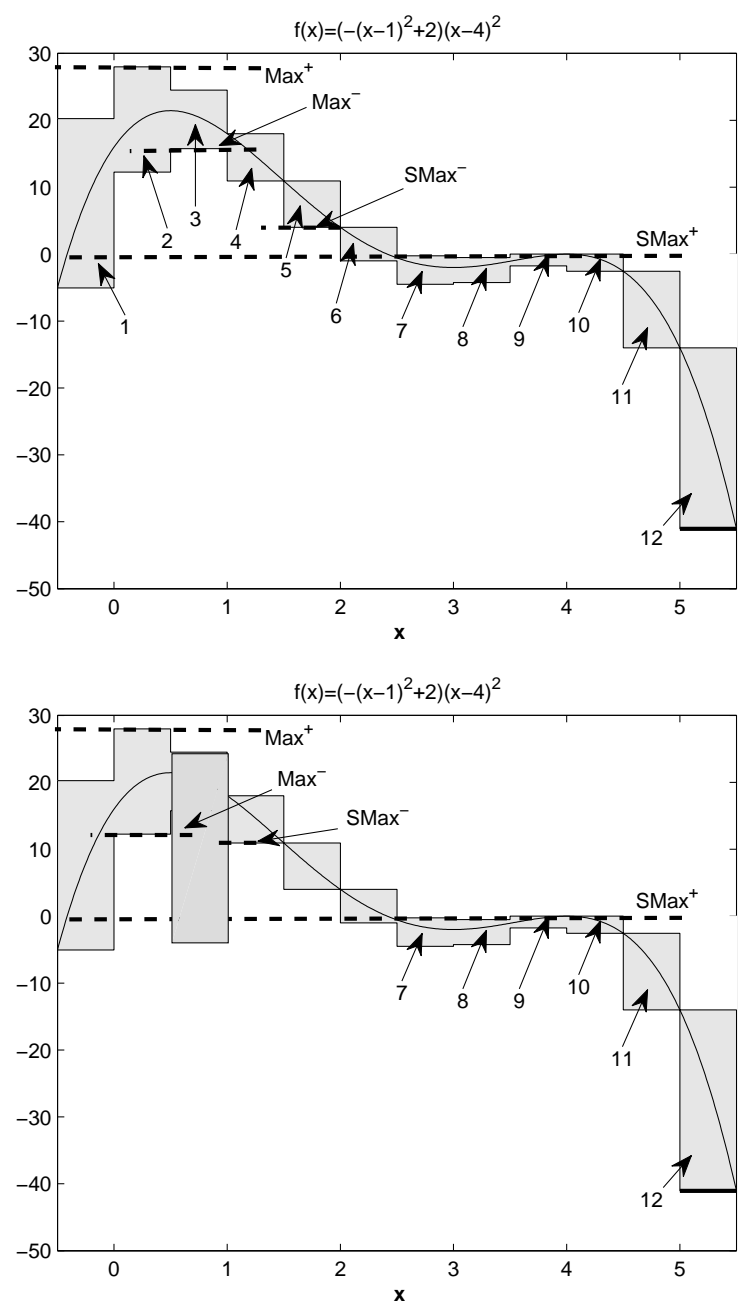

FIGURE 3. Connected and non connected sets.

with its complementary image. Since $\{\mathcal{L}\}_{[f],\{\mathcal{P}\}, a}^{+}$is supposed to be connected, it constitutes a SMidentifiable set.

Similarly, one gets the following proposition.

\section{Proposition 3.5.}

In (3.3), for $a=\mathcal{S} \mathcal{M i n}_{[f],\{\mathcal{P}\}^{-}}$, if the list $\{\mathcal{L}\}_{[f],\{\mathcal{P}\}, a}^{-}$is a connected set, then it is a SM-identifiable set. We denote it by $\{\mathcal{L}\}_{S M I,[f],\{\mathcal{P}\}}^{-}$.

Figure 3 providing by Equation (3.1) illustrates the importance of the connected property verification.

In the first Figure, one gets $a=\mathcal{S} \mathcal{M a x}{ }_{[f],\{\mathcal{P}\}^{+}}=\max \left([f]\left(\{\mathcal{P}\}_{7}\right)\right)$, $\{\mathcal{L}\}_{[f],\{\mathcal{P}\}, a}^{+}=\left\{\{\mathcal{P}\}_{2},\{\mathcal{P}\}_{3},\{\mathcal{P}\}_{4},\{\mathcal{P}\}_{5}\right\}$ and it can be easily seen that $\{\mathcal{L}\}_{[f],\{\mathcal{P}\}, a}^{+}$is the list $\{\mathcal{L}\}_{S M I,[f],\{\mathcal{P}\}}^{+}$since it is connected. Notice that $\mathcal{S} \mathcal{M a x}_{[f],\{\mathcal{P}\}}{ }^{-}$is the lower bound of a sub-list. However, in the second Figure, $\{\mathcal{L}\}_{[f],\{\mathcal{P}\}, a}^{+}=\left\{\{\mathcal{P}\}_{2},\{\mathcal{P}\}_{4},\{\mathcal{P}\}_{5}\right\}$ is no more connected and can 
not be a SM-identifiable set.

Algorithm 2 is used to find the connected sets verifying Proposition 3.4. In this algorithm, two lists $\left\{\mathcal{L}_{1}\right\}$ and $\left\{\mathcal{L}_{2}\right\}$ verify $\left\{\mathcal{L}_{1}\right\}=\left\{\mathcal{L}_{2}\right\}$, if they are composed of the same elements even in different order. Moreover, the notation $\left[\mathbf{x}_{a}\right]$ designates the component $\{\mathcal{P}\}_{i}$ of the list $\{\mathcal{P}\}$ for which $\min \left([f]\left(\{\mathcal{P}\}_{i}\right)\right)=a$.

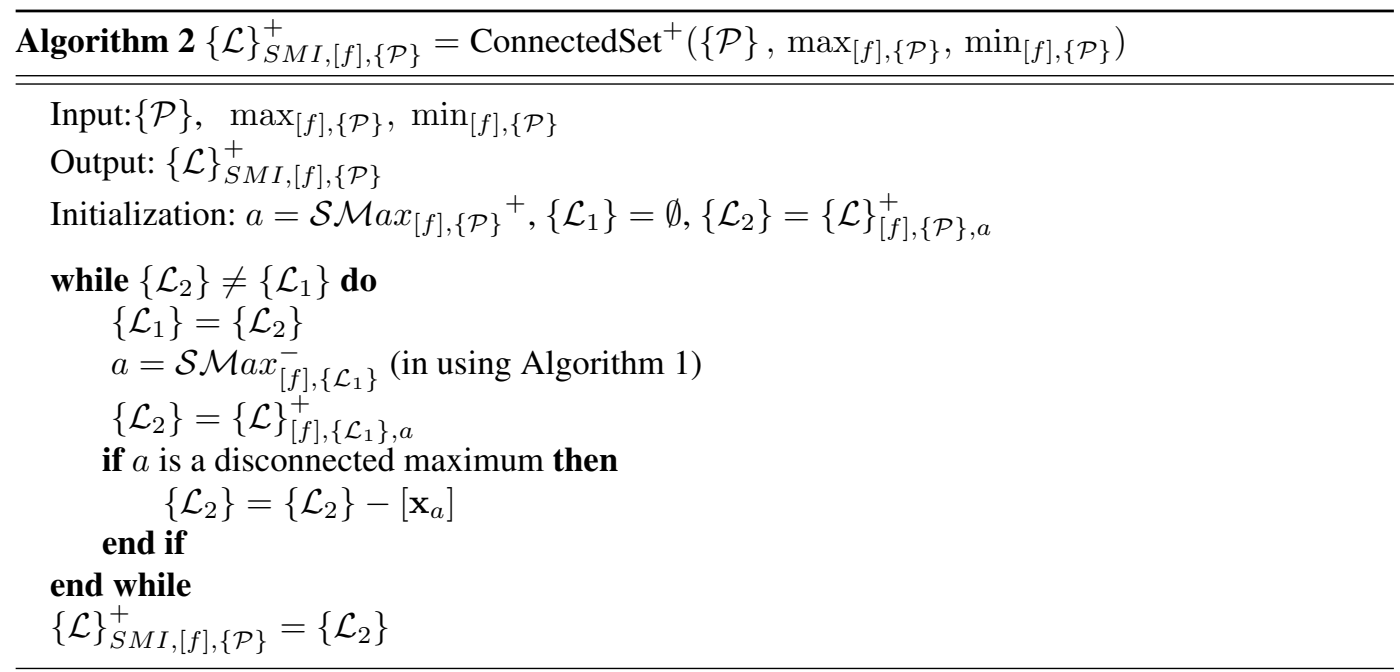

Another algorithm can be deduced in substituting + and max by - and min in order to find $\{\mathcal{L}\}_{S M I,[f],\{\mathcal{P}\}}^{-}$

3.1.3. Final algorithms. Algorithms 3 and 4 are the final algorithms which determine the SMidentifiable sets in the definition domain of a function and take again the previous Algorithms 1 and 2. $\{\mathcal{U}\}_{S M I,[f],\{\mathcal{P}\}}$ corresponds to the set of the two lists $\{\mathcal{L}\}_{S M I,[f],\{\mathcal{P}\}}^{+}$and $\{\mathcal{L}\}_{S M I,[f],\{\mathcal{P}\}}^{-}$.

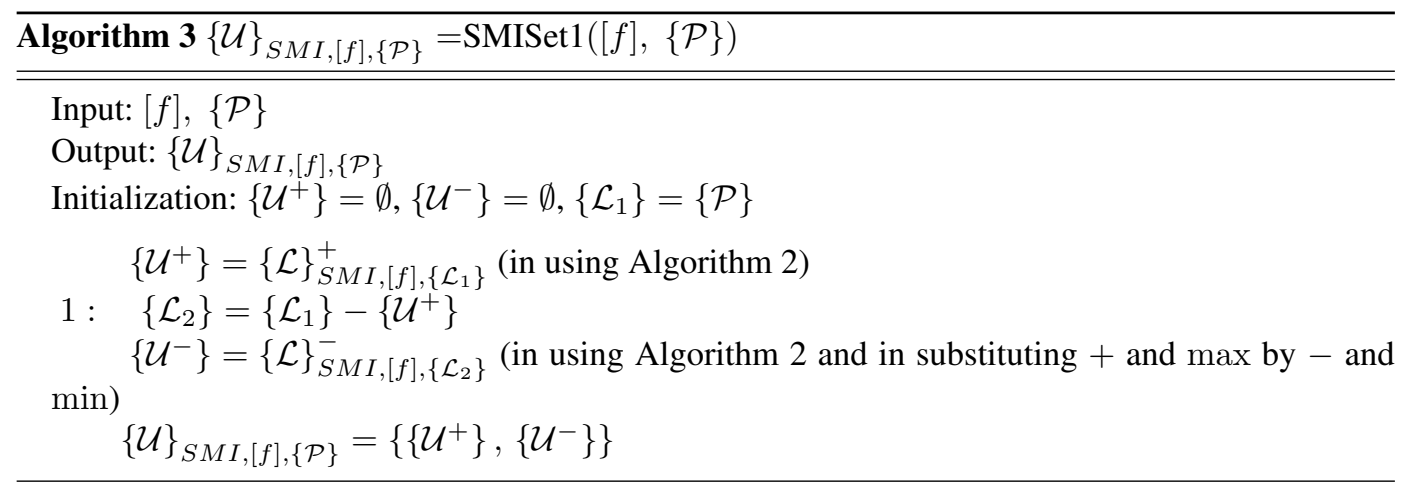




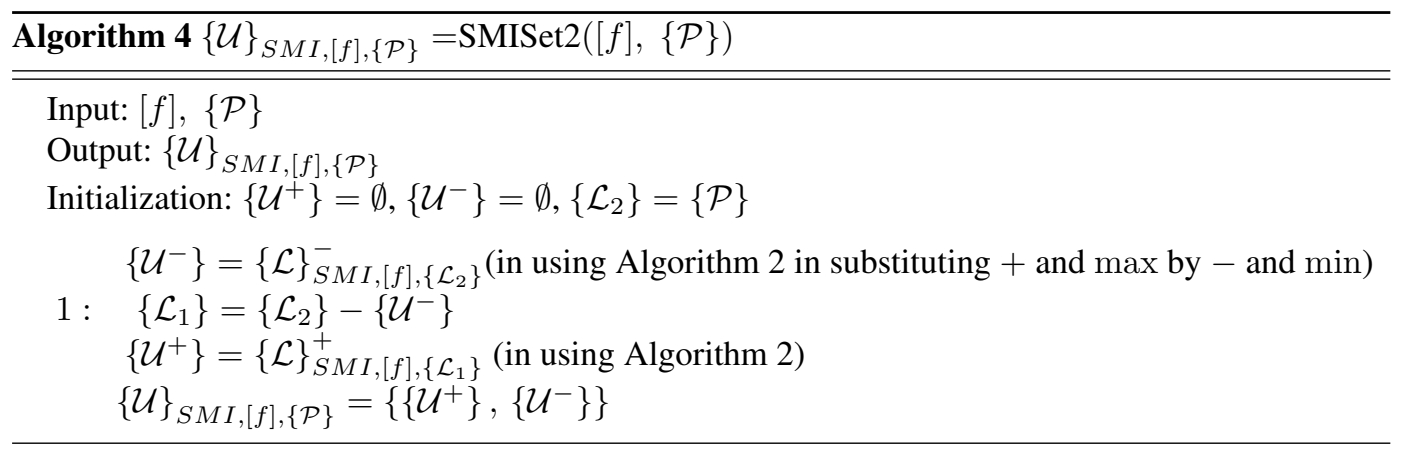

As it can be seen, these algorithms are different only in the order of treating the partitions $\left\{\mathcal{U}^{+}\right\}$ and $\left\{\mathcal{U}^{-}\right\}$. In addition, line 1 , consisting in the subtraction of two lists, prevents the apparition of the same boxes in $\left\{\mathcal{U}^{+}\right\}$and $\left\{\mathcal{U}^{-}\right\}$.

\subsection{The case of multidimensional functions}

In this section, we consider the case $m>1$. We suppose that an interval vector function $[f]=$ $\left[\left[f_{1}\right], \ldots,\left[f_{m}\right]\right]$ and a subpaving $\{\mathcal{P}\}$ of $[x]$ are available. The main idea of Algorithm 5 consists in applying Algorithms 3 or 4 consecutively on each component of the vector $[f]$. For example, Algorithm 3 is applied with $\left[f_{1}\right]$ and the list $\{\mathcal{P}\}$. Suppose that it gives the list $\{\mathcal{U}\}_{S M I,\left[f_{1}\right],\{\mathcal{P}\}}$ of SM-identifiable sets. Algorithm 3 is then reapplied with $\left[f_{2}\right]$ and $\{\mathcal{P}\}-\{\mathcal{U}\}_{S M I,\left[f_{1}\right],\{\mathcal{P}\}}$. The process goes on until the last component of the function $[f]$.

Based on Algorithms 3 and 4, Agorithm 5 has been implemented.

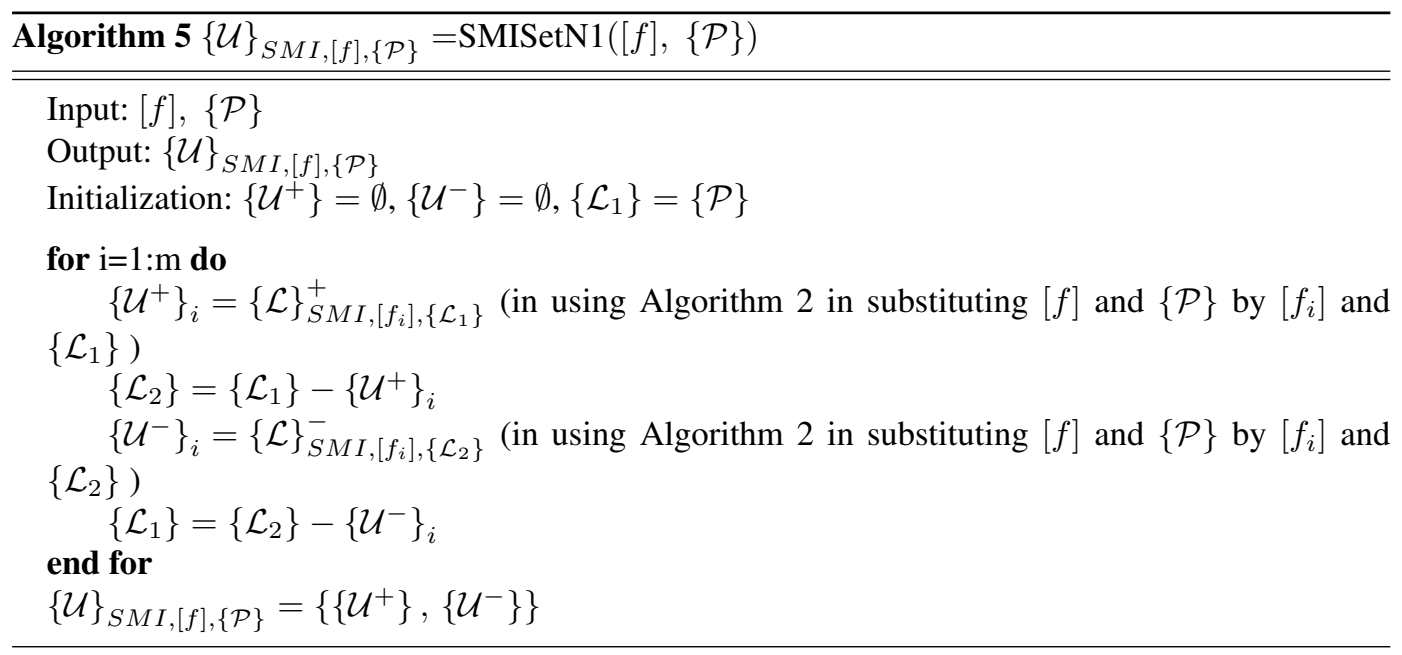

However, as in the case of $m=1$, other algorithms can be considered, for example the following algorithm: 

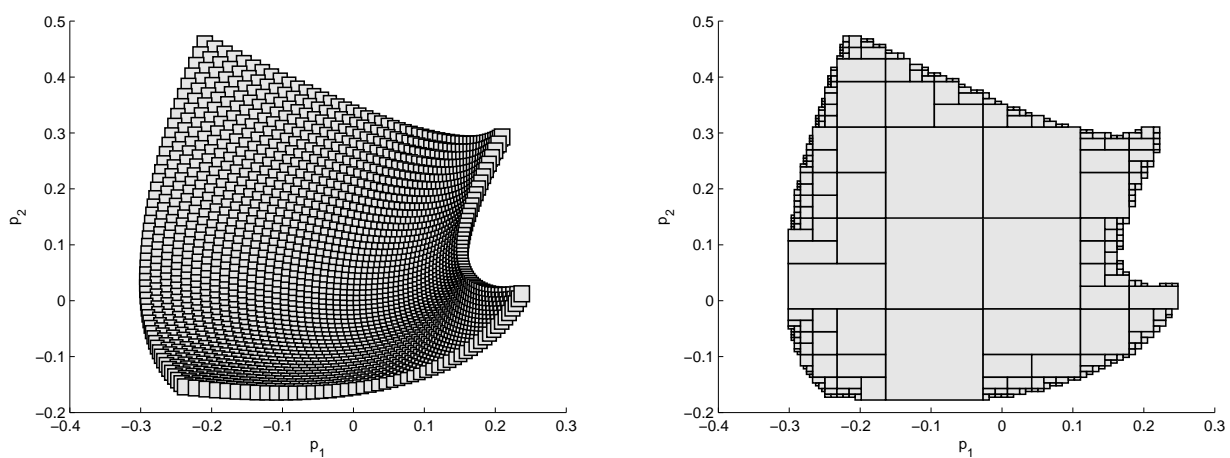

FIGURE 4. (a): list of overlapped boxes , (b): external regular subpaving

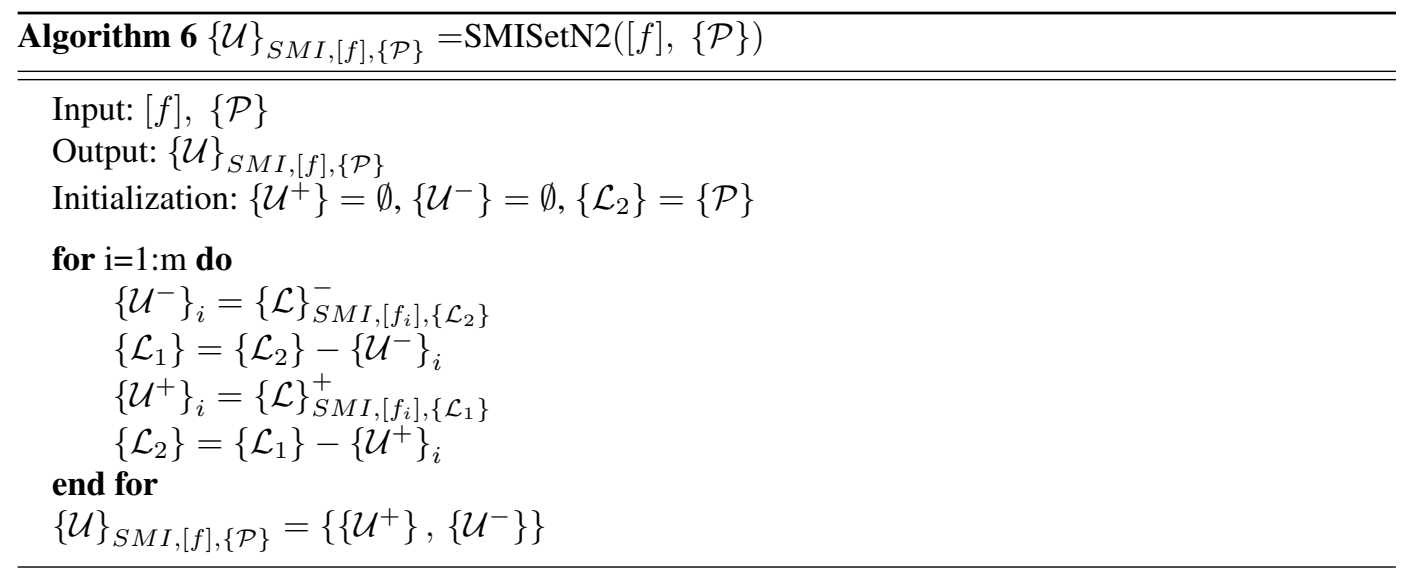

Changing the order of the interval functions, $\left[f_{i}\right], i=1, \ldots, m$ and also the order of computing partitions $\left\{\mathcal{U}^{-}\right\}_{i}$ and $\left\{\mathcal{U}^{+}\right\}_{i}$, altogether, there can be $(2 m)$ ! algorithms like Algorithms 5 and 6 .

\subsection{Regularization}

In [6], in order to evaluate the image of a function at a domain set, three main sequential steps, mince, evaluate and regularize are introduced. In Sections 3.3 and 3.4, the two first steps, the mince and the evaluate ones have been explained. In this section, the last step, i.e. the regularization is realized. Figure 4 illustrates an example of the regularization step based on the algorithm presented in [6]. This algorithm can also be applied to a list composing of overlapped or non overlapped boxes. However, as it can be seen in Figure 4, the initial list, here consisting of overlapped boxes in Figure 4 (a), is inside the regular subpaving of the Figure 4 (b), or, in other words, the subpaving is an external regular subpaving. If we apply this algorithm to a list of SM-identifiable sets, some parts of the initial domain which are not SM-identifiable will be included in the obtained regular subpaving. To cope with this problem, the Algorithm 7 is proposed to find a regular subpaving inside a list. However, the list must only consist of non overlapped boxes which is fortunately the case of a list of SM-identifiable sets found by the method presented in the previous sections.

In this algorithm, the function named volume and applied to an interval box $[\mathbf{x}]=\left[\left[\mathbf{x}_{1}\right]\left[\mathbf{x}_{2}\right] \ldots\left[\mathbf{x}_{n}\right]\right]^{T} \in$ $\mathbb{I R}^{n}$ is defined as: volume $([\mathbf{x}])=\left(\overline{\mathbf{x}}_{1}-\underline{\mathbf{x}}_{1}\right) \times\left(\overline{\mathbf{x}}_{2}-\underline{\mathbf{x}}_{2}\right) \times \ldots \times\left(\overline{\mathbf{x}}_{n}-\underline{\mathbf{x}}_{n}\right)$ with $\left[\mathbf{x}_{i}\right]=\left[\underline{\mathbf{x}}_{i}, \overline{\mathbf{x}}_{i}\right]$ for $i=1, \ldots, n$.

To explain how this algorithm works, we consider the simple case $n=2$. In this case, since $[\mathbf{x}]$ is a rectangle, the function volume consists in evaluating its surface. Beginning by the convex 


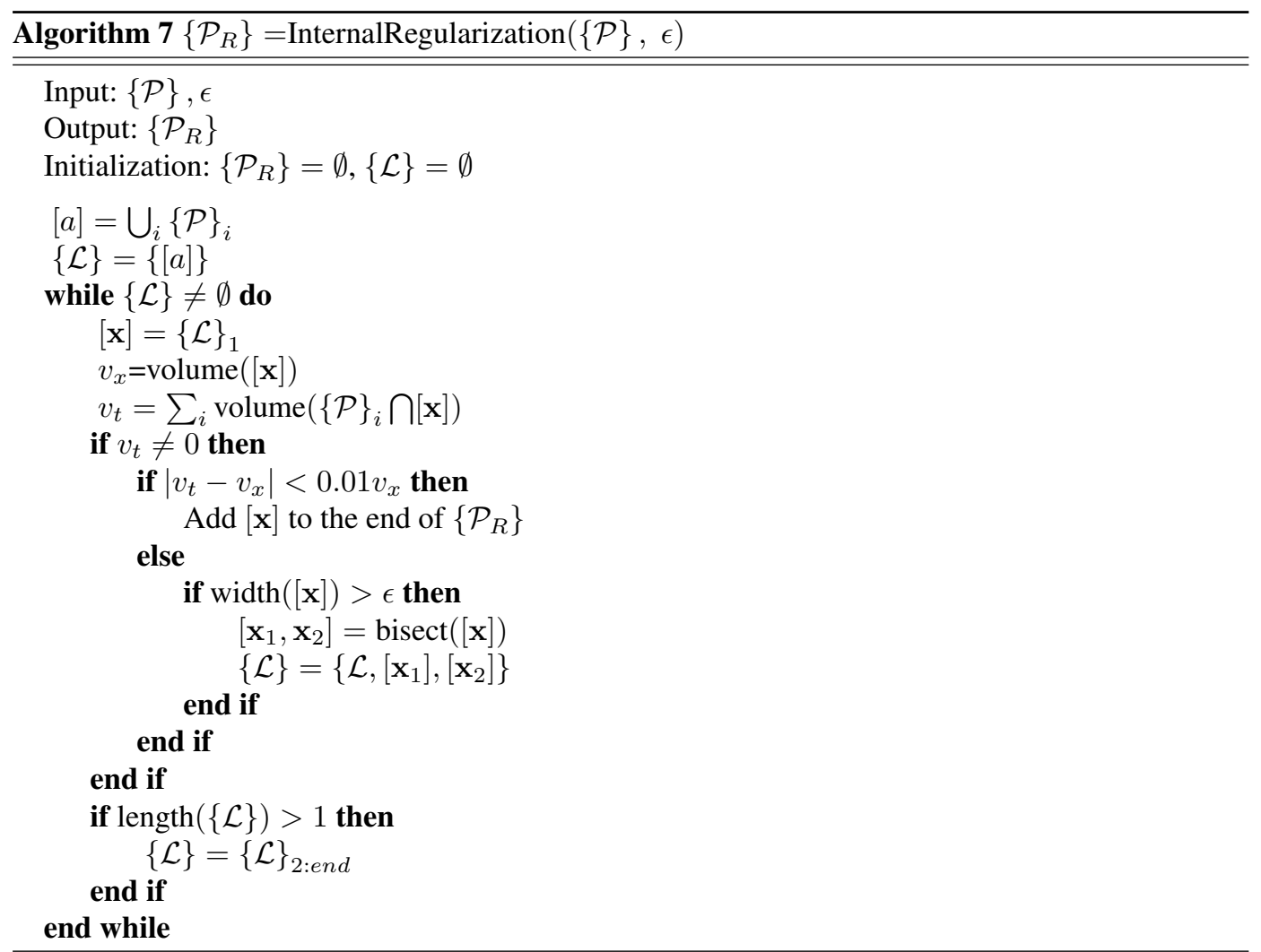

union of the paving $\{\mathcal{P}\}$, we construct a list by consecutive bisections as explained in that follows. At each iteration, we get the first element of the last list, i.e. $[\mathbf{x}]$, and we evaluate its surface, i.e. $v_{x}$. We compute also the surface of this element which is covered by the paving, i.e. $v_{t}$. If $v_{t}$ exists, and if its difference with $v_{x}$ is very small comparing to $v_{x}$, then $[\mathbf{x}]$ is accepted and is added to the response list $\left\{\mathcal{P}_{R}\right\}$. If $v_{x}$ exists but the difference is large and $[\mathbf{x}]$ is wide compared to the precision $\epsilon$, then we bisect $[\mathbf{x}]$ and we add the results to the end of the last list. The new list is produced. Recall that the elements of $\{\mathcal{P}\}$ do not intersect and consequently $\{\mathcal{P}\}_{i} \cap[\mathbf{x}]$ do not have intersection with $\{\mathcal{P}\}_{j} \cap[\mathbf{x}]$ for all $i, j$ from 1 to length $(\{\mathcal{P}\}), i \neq j$. This property enables us to compare sum of the surfaces $\{\mathcal{P}\}_{i} \cap[\mathbf{x}]$, i.e. $v_{t}$, with the surface of $[\mathbf{x}]$, i.e. $v_{x}$.

Figure 5 shows the difference between internal and external regular subpavings found for a list. As it can be seen, the list consists of non overlapped boxes (Figure 8 (a)), the internal regular subpaving is inside the list (Figure 8 (b)), and the external regular subpaving is outside the list (Figure $8(\mathrm{c}))$.

\section{Examples}

Example 2: Consider the Bernoulli equation $\dot{y}(t)=-p_{1}^{2} y(t)+p_{2}^{2} y(t)^{2}$ and let us applied the steps proposed at section 2.4 .

1. We have directly

$$
w(y, p)=\dot{y}(t)+p_{1}^{2} y(t)-p_{2}^{2} y(t)^{2} .
$$



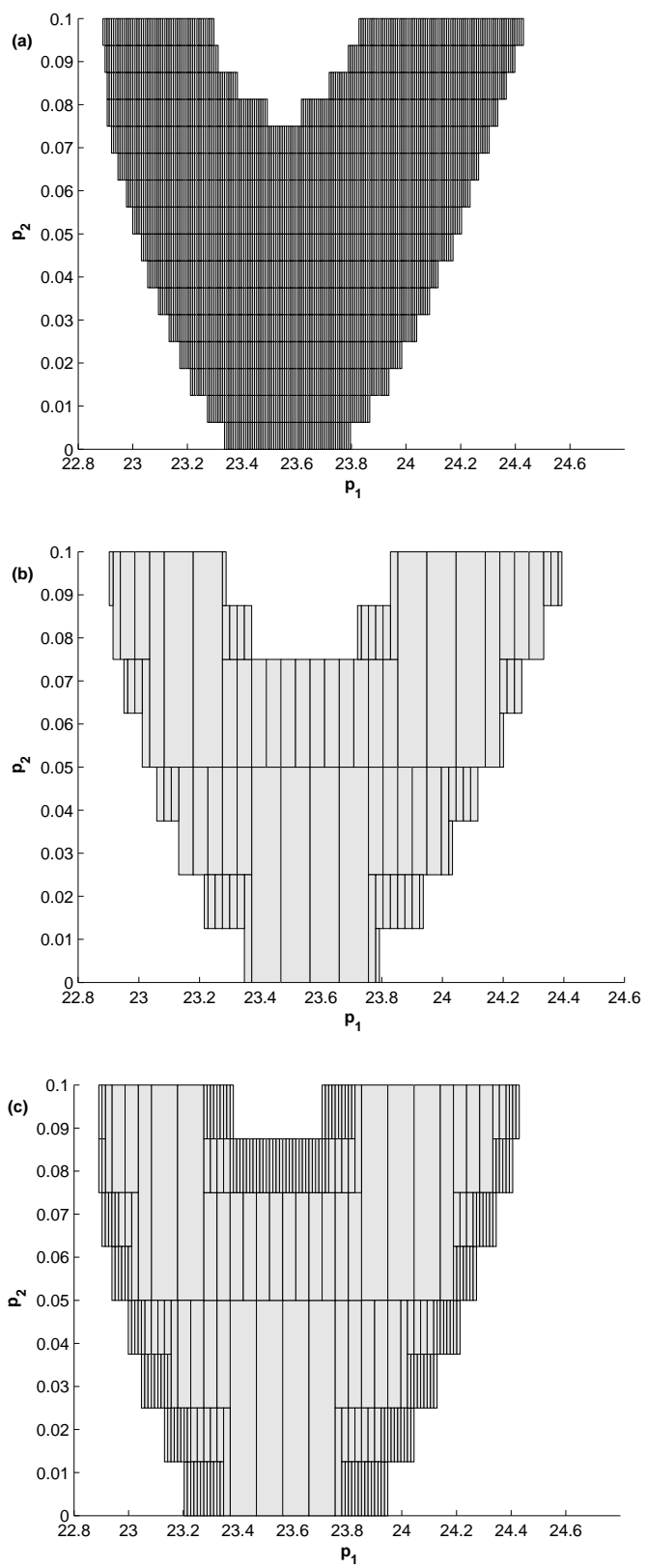

FIGURE 5. (a): list of non overlapped boxes, (b): internal regular subpaving (c): external regular subpaving

2. Its functional determinant is equal to $\dot{y} y^{2}$ which is not identically equal to zero.

In that case, the function $\phi$ is defined by $\phi: \mathbb{R}^{2} \rightarrow \mathbb{R}^{2}$, $\phi(p)=\left[\begin{array}{l}p_{1}^{2} \\ -p_{2}^{2}\end{array}\right]$

3. Algorithm 5 is used with a regular paving such that its resolution is $\epsilon=0.05$. The results are illustrated in Figure 6 after regularization. Light gray, dark gray and black color boxes 
correspond to SM-identifiable sets but which are not $\mu$ SM-identifiable. The state gray color box represents the $\mu$-SM-identifiable sets.

With Algorithm 6, we do not obtain such SM-identifiable sets.

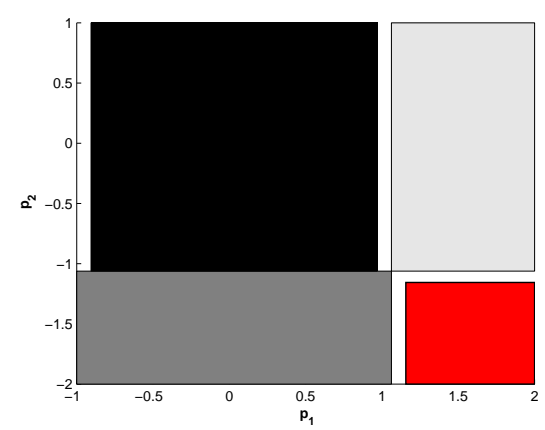

FIGURE 6. Example 2: State gray: $\mu$-SM-identifiable set, light Grays and Black: SM-identifiable sets.

Example 3: Consider the following example:

$$
\left\{\begin{array}{l}
\dot{x}_{1}(t)=x_{1}(t)+x_{2}(t)+u(t), \quad x_{1}(0)=p_{1}\left(1+\sin \left(p_{1}\right)-p_{2} \sin \left(p_{1}\right)\right)+p_{2} \cos \left(p_{1}\right), \\
\dot{x}_{2}(t)=-x_{1}^{2}(t)-\left(\left(1-p_{2}\right) p_{1} \cos \left(p_{1}\right)-p_{2} \sin \left(p_{1}\right)-2 p_{2}\right) x_{1} x_{2}, x_{2}(0)=0, \\
y=x_{1}
\end{array}\right.
$$

1. By setting $c_{1}=\cos \left(p_{1}\right)$ and $c_{2}=\sin \left(p_{1}\right)$, the Rosenfeld-Groebner algorithm, implemented in the package DifferentialAlgebra of Maple gives the following differential polynomial:

$w(y, u, p)=-\dot{u}-\dot{y}+\ddot{y}+y^{2}+\left(\left(1-p_{2}\right) p_{1} \cos \left(p_{1}\right)-p_{2} \sin \left(p_{1}\right)-2 p_{2}\right)\left(\dot{y} y-u y-y^{2}\right)$.

2. Clearly the associated functional determinant is not identically equal to zero. Suppose too that $u(0)=0$, then the following function $\phi: \mathbb{R}^{2} \rightarrow \mathbb{R}^{2}$ can be considered:

$\phi(p)=\left[\begin{array}{l}\left(1-p_{2}\right) p_{1} \cos \left(p_{1}\right)-p_{2} \sin \left(p_{1}\right)-2 p_{2} \\ p_{1}\left(1+\sin \left(p_{1}\right)-p_{2} \sin \left(p_{1}\right)\right)+p_{2} \cos \left(p_{1}\right)\end{array}\right]$.

3. Choosing $\epsilon=0.01$ and Algorithm 5, the results illustrated in Figure 7 are obtained.

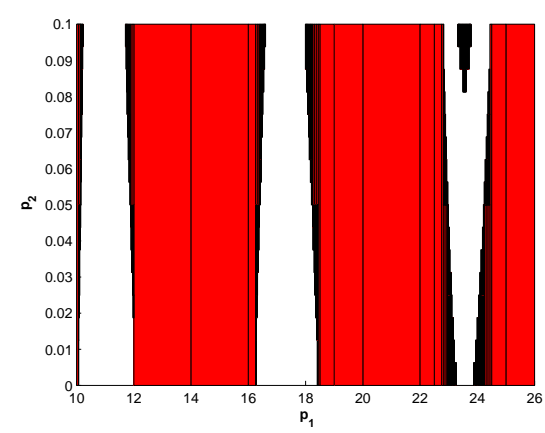

FIGURE 7. Example 3: State gray: $\mu$-SM-identifiable sets, Black: SM-identifiable sets. 


\section{Conclusion}

In this paper, we propose a guaranteed method in order to determine, in the parameter space of a model, the $(\mu$-)SM-identifiable sets, each of them corresponding to different output behaviors from their complementary. For doing this, differential algebra tools have been used for obtaining differential polynomials. From them, a real function depending only on the parameters has been constructed. We show that the domains on which this function is partially injective correspond to $\mu$ SM-identifiable sets, otherwise if the function verifies only the restricted-partial injectivity, the corresponding domain determines SM-identifiable sets. An algorithm based on interval analysis tools has been proposed to determine the SM-identifiable sets in the function's range, the latter corresponding to parameter space. Finally, Two examples have been provided in order to study the SM-identifibility of the model.

\section{References}

[1] N. Bourbaki. Elements of Mathematics. Springer-Verlag, 1989.

[2] I. Braems, L. Jaulin, M. Kieffer, and E. Walter. Guaranteed numerical alternatives to structural identiability testing. In Proceedings of the 40th IEEE CDC, pages 3122-3127, Orlando, USA, 2001.

[3] G. Chabert and L. Jaulin. Contractor programming. Artifical Intelligence, 173:1079-1100, 2009.

[4] C. Jauberthie, N. Verdière, and L. Travé-Massuyès. Set-membership identifiability: definitions and analysis. In Proceedings of the 18th IFAC World Congress, pages 12024-12029, Milan, Italie, 2011.

[5] C. Jauberthie, N. Verdière, and L. Travé-Massuyès. Fault detection and identification relying on setmembership identifiability. Annual Reviews in Control, 73(1):129-136, 2013.

[6] L. Jaulin, M. Kieffer, O. Didrit, and E. Walter. Applied Interval Analysis, with examples in parameter and state estimation, Robust control and robotics. Springer, Londres, 2001.

[7] E.R. Kolchin. Differential algebra and algebraic groups. Academic Press, New York, 1973.

[8] S. Lagrange, N. Delanoue, and L. Jaulin. On sufficient conditions of injectivity, development of a numerical test via interval analysis. Journal Reliable computing, 13:409-421, 2007.

[9] S. Lagrange, N. Delanoue, and L. Jaulin. Injectivity analysis using interval analysis : Application to structural identifiability. Automatica, 44 (11):2959-2962, 2008.

[10] L. Ljung and T. Glad. On global identifiability for arbitrary model parametrizations. Automatica, 30:265276, 1994.

[11] M. Milanese and A. Vicino. Estimation theory for nonlinear models and set membership uncertainty. Automatica, 27(2):403-408, 1991.

[12] J. R. Munkres. Topology a first courses. Prentice Hall, New Jersey, 1975.

[13] T. Rasssi, N. Ramdani, and Y. Candau. Set-membership state and parameter estimation for systems described by nonlinear differential equations. Automatica, 40(10):1771-1777, 2004.

[14] N. Verdière, L. Denis-Vidal, G. Joly-Blanchard, and D. Domurado. Identifiability and estimation of pharmacokinetic parameters of ligands of macrophage mannose receptor. Int. J. Appl. Math. Comput. Sci, 15:101-110, 2005.

[15] N. Verdière, C. Jauberthie, and L. Travé-Massuyès. Set-membership identifiability of nonlinear models. LAAS Report, Number 13001:8 pages, 2013.

[16] E. Walter, J. Norton, H. H. Piet-Lahanier, and M. Milanese. Bounding Approaches to System Identification. Perseus Publishing, 1996.

[17] E. Walter and H. Piet-Lahanier. Estimation of parameter bounds from bounded-error data: a survey. Mathematics and Computers in simulation, 32(5):449-468, 1990. 


\section{Acknowledgment}

This work was supported by the French National Research Agency (ANR) in the framework of the project ANR-11-INSE-006 (MAGIC-SPS).

Laleh Ravanbod

Université Paul Sabatier,

Institut de Mathématiques de Toulouse,

118 route de Narbonne,

F-31062 Toulouse Cedex 9.

e-mail: laleh.hosseini@math.univ-toulouse.fr

Nathalie Verdière

Université du Havre,

25 rue Philippe Lebon,

BP 1123, 76063 Le Havre Cedex,

France.

e-mail: verdiern@univ-lehavre.fr

Carine Jauberthie

CNRS, LAAS, 7 avenue du Colonel Roche, F-31400 Toulouse, France;

Université de Toulouse, UPS, LAAS, F-31400 Toulouse, France.

e-mail: cjaubertelaas.fr 